\title{
Zeta Value Identities From Iterated Integrals With Additional Factors
}

\author{
Chan-Liang Chung ${ }^{1} \&$ Minking Eie ${ }^{2}$ \\ ${ }^{1}$ College of Mathematics and Computer Science, Fuzhou University, Fuzhou, China \\ ${ }^{2}$ Department of Mathematics, National Chung Cheng University, Chiayi, Taiwan (R.O.C.) \\ Correspondence: Chan-Liang Chung, College of Mathematics and Computer Science, Fuzhou University, Fuzhou, China. \\ E-mail: andrechung@fzu.edu.cn
}

Received: July 1, 2019 Accepted: September 2, 2019 Online Published: September 15, 2019

doi:10.5539/jmr.v11n5p40 URL: https://doi.org/10.5539/jmr.v11n5p40

\begin{abstract}
A multiple zeta value can always be represented by its Drinfel'd integral. If we add some factors appeared in the integrand of the integral representation of the multiple zeta value, it would still represent a linear combination of multiple zeta values, but the depths and weights may decrease. In this paper, we shall investigate some of multiple zeta values obtained from Drinfel'd integral with additional factors aforementioned and study a class of deformation of multiple zeta values. Results are then obtained as analogues or generalizations of the sum formula of multiple zeta values.
\end{abstract}

Keywords: multiple zeta value, Drinfel'd integral, sum formula, duality, Euler sum with two branches

\section{Introduction}

A multiple zeta value (resp. multiple zeta star value) is defined as (see (J. M. Borwein, D. M. Bradley, Broadhurst \& Lisoněk, 2001), or (Eie, 2009))

$$
\begin{gathered}
\zeta(\boldsymbol{\alpha})=\zeta\left(\alpha_{1}, \alpha_{2}, \ldots, \alpha_{r}\right)=\sum_{1 \leq k_{1}<k_{2}<\cdots<k_{r}} k_{1}^{-\alpha_{1}} k_{2}^{-\alpha_{2}} \cdots k_{r}^{-\alpha_{r}} \\
\text { resp. } \left.\quad \zeta^{\star}(\boldsymbol{\alpha})=\zeta^{\star}\left(\alpha_{1}, \alpha_{2}, \ldots, \alpha_{r}\right)=\sum_{1 \leq k_{1} \leq k_{2} \leq \cdots \leq k_{r}} k_{1}^{-\alpha_{1}} k_{2}^{-\alpha_{2}} \cdots k_{r}^{-\alpha_{r}}\right),
\end{gathered}
$$

with positive integers $\alpha_{1}, \alpha_{2}, \ldots, \alpha_{r}$ and $\alpha_{r} \geq 2$ for the sake of convergence. The number $r$ and $|\boldsymbol{\alpha}|=\alpha_{1}+\alpha_{2}+\cdots+\alpha_{r}$ are the depth and the weight of the multiple zeta value, respectively.

Due to Kontsevich (Drinfel'd, 1991), multiple zeta values can be represented by iterated integrals or Drinfel'd integrals over simplices of weight dimension:

$$
\zeta\left(\alpha_{1}, \alpha_{2}, \ldots, \alpha_{r}\right)=\int_{E_{|\alpha|}} \Omega_{1} \Omega_{2} \cdots \Omega_{|\alpha|}
$$

with $E_{|\alpha|}: 0<t_{1}<t_{2}<\cdots<t_{|\alpha|}<1$ and

$$
\Omega_{j}= \begin{cases}\frac{d t_{j}}{1-t_{j}} & \text { if } j=1, \alpha_{1}+1, \alpha_{1}+\alpha_{2}+1, \ldots, \alpha_{1}+\alpha_{2}+\ldots+\alpha_{r-1}+1 ; \\ \frac{d t_{j}}{t_{j}} & \text { otherwise. }\end{cases}
$$

We say that $\Omega_{j}$ is of type one if $\Omega_{j}=d t_{j} /\left(1-t_{j}\right)$, and is of type two if $\Omega_{j}=d t_{j} / t_{j}$. Let $u_{j}=1-t_{|\alpha|+1-j}$ for $j=1,2, \ldots,|\alpha|$. We have $0<u_{1}<u_{2}<\cdots<u_{|\alpha|}<1$ and the dual of $\zeta(\alpha)$,

$$
\zeta\left(\boldsymbol{\alpha}^{\vee}\right)=\int_{0<u_{1}<u_{2}<\cdots<u_{|\alpha|}<1} \omega_{1} \omega_{2} \cdots \omega_{|\alpha|},
$$

where

$$
\omega_{j}= \begin{cases}\frac{d u_{j}}{1-u_{j}} & \text { if } \Omega_{|\alpha|+1-j} \text { is of type one; } \\ \frac{d u_{j}}{u_{j}} & \text { if } \Omega_{|\alpha|+1-j} \text { is of type two. }\end{cases}
$$

For some $j$, if we add the factor $t_{j}^{b}$ or $\left(1-t_{j}\right)^{b}$ to the iterated integral representations of multiple zeta values, it would lead to the change of the depth and weight, but still represent a linear combination of multiple zeta values. For example,

$$
\zeta(3)=\int_{E_{3}} \frac{d t_{1}}{1-t_{1}} \frac{d t_{2}}{t_{2}} \frac{d t_{3}}{t_{3}} .
$$


If we add the factor $t_{1}$, it becomes

$$
\int_{E_{3}} \frac{t_{1} d t_{1}}{1-t_{1}} \frac{d t_{2}}{t_{2}} \frac{d t_{3}}{t_{3}}
$$

and it can be evaluated as

$$
\sum_{k=1}^{\infty} \frac{1}{(k+1)^{3}}=\zeta(3)-1 \text {. }
$$

Now we add the factor $t_{3}$ to the integral representation of $\zeta(3)$, so

$$
\int_{E_{3}} \frac{d t_{1}}{1-t_{1}} \frac{d t_{2}}{t_{2}} d t_{3}
$$

represents the series

$$
\sum_{k=1}^{\infty} \frac{1}{k^{2}(k+1)}
$$

which is equal to $\zeta(2)-1$. In general, if we replace some of $d t_{j} /\left(1-t_{j}\right)$ or $d t_{j} / t_{j}$ in the iterated integral representation of multiple zeta values by $d t_{j}$, the deformed iterated integral still represent a linear combination of multiple zeta values, but their depths and weights may decrease.

As another illustrated example, let $p$ and $q$ be two positive integers, the multiple zeta value $\zeta\left(\{1\}^{p-1}, q+1\right)$ has the iterated integral repression

$$
\zeta\left(\{1\}^{p-1}, q+1\right)=\int_{E_{p+q}}\left(\prod_{i=1}^{p} \frac{d t_{i}}{1-t_{i}}\right)\left(\prod_{j=p+1}^{p+q} \frac{d t_{j}}{t_{j}}\right) .
$$

If we add the factor $t_{p+1} t_{p+2} \cdots t_{p+q}$ to the iterated integral, it becomes

$$
\int_{E_{p+q}}\left(\prod_{i=1}^{p} \frac{d t_{i}}{1-t_{i}}\right)\left(\prod_{j=p+1}^{p+q} d t_{j}\right)
$$

and can be evaluated easily as

$$
\sum_{1 \leq k_{1}<k_{2}<\cdots<k_{p}} \frac{1}{k_{1} k_{2} \cdots k_{p}\left(k_{p}+1\right)\left(k_{p}+2\right) \cdots\left(k_{p}+q\right)} .
$$

Also the dual of the iterated integral is given by

$$
\int_{E_{q+p}}\left(\prod_{j=1}^{q} d u_{j}\right)\left(\prod_{i=q+1}^{q+p} \frac{d u_{i}}{u_{i}}\right)
$$

which has the value $\frac{1}{q^{p}(q !)}$. Of course, we have the identity

$$
\sum_{1 \leq k_{1}<k_{2}<\cdots<k_{p}} \frac{1}{k_{1} k_{2} \cdots k_{p}\left(k_{p}+1\right)\left(k_{p}+2\right) \cdots\left(k_{p}+q\right)}=\frac{1}{q^{p}(q !)} .
$$

The following result is of similar fashion.

Proposition 1.1 [see Proposition 4.1, (Chen, Chung \& Eie, 2016)] Suppose that $\zeta\left(\alpha_{1}, \ldots, \alpha_{r-1}, \alpha_{r}+1\right)$ is a multiple zeta value of depth $r$ and weight $w(\geq r+1)$ with the iterated integral representation $\int_{E_{w}} \Omega_{1} \Omega_{2} \cdots \Omega_{w}$. Then for real numbers $a, b>-1$, we have

$$
\int_{E_{w}}\left(\frac{t_{1}}{t_{w}}\right)^{a} \Omega_{1} \Omega_{2} \cdots \Omega_{w}=\sum_{1 \leq \ell_{1}<\ell_{2}<\cdots<\ell_{r}}\left(\ell_{1}+a\right)^{-\alpha_{1}}\left(\ell_{2}+a\right)^{-\alpha_{2}} \cdots\left(\ell_{r}+a\right)^{-\alpha_{r}} \ell_{r}^{-1},
$$

and

$$
\begin{aligned}
& \int_{E_{w}}\left(\frac{1-u_{w}}{1-u_{1}}\right)^{b} \Omega_{1} \Omega_{2} \cdots \Omega_{w} \\
= & \sum_{1 \leq k_{1}<k_{2}<\cdots<k_{r}} k_{1}^{-\alpha_{1}} k_{2}^{-\alpha_{2}} \cdots k_{r}^{-\alpha_{r}-1} \frac{\Gamma\left(k_{1}+b\right) \Gamma\left(k_{r}+1\right)}{\Gamma\left(k_{1}\right) \Gamma\left(k_{r}+1+b\right)},
\end{aligned}
$$


where $\Gamma(s)$ is the gamma function.

Multiple zeta values with parameters were first introduced in (Chen, Chung \& Eie, 2016; Eie \& Lee, 2016) in order to provide a simple way to reprove the sum formula as well as the restricted sum formula (Eie, Liaw \& Ong, 2009). Also they provided a systematic way to evaluate iterated integrals with parameters.

In this paper, we are going to investigate some zeta values obtained from Drinfel'd integrals with additional factors. In particular, for a real $a>-1$ and nonnegative integers $p, r$ and $q$ with $r+q \geq 1$, we consider the iterated integral

$$
I_{p, r, q}(a)=\int_{E_{p+r+q}}\left(\frac{t_{1}}{t_{p+r+q}}\right)^{a}\left(\prod_{i=1}^{p} \frac{d t_{j}}{1-t_{j}}\right)\left(\prod_{j=p+1}^{p+r} d t_{j}\right)\left(\prod_{k=p+r+1}^{p+r+q} \frac{d t_{k}}{t_{k}}\right) .
$$

In Section 2, along with multiple zeta values with parameters, we give the relation between $I_{p, r, q}(a)$ and its dual when $q=0$ and produce some analogues of the sum formula. In Section 3, we force on the evaluation of $I_{p, r, q}(a)$ when $q=1$ and $q \geq 2$ respectively and generalize the well-known sum formula from this point of view and obtain some transforms of weighted sum formulas which are difficult to be obtained otherwise. Indeed, we derive the following result.

To state our further results more precisely, we explain some notations first. We write $\lambda \vdash n$ to denote that $\lambda=\left(\lambda_{1}, \lambda_{2}, \ldots, \lambda_{g}\right)$ is a partition of $n$, i.e., $1 \leq \lambda_{1} \leq \lambda_{2} \leq \cdots \leq \lambda_{g}$ and $|\lambda|=\lambda_{1}+\lambda_{2}+\cdots+\lambda_{g}=n$. Also we let $\{a\}^{m}$ be the $m$ repetitions of $a$. When $\lambda=\left(\{1\}^{m_{1}},\{2\}^{m_{2}}, \ldots,\{k\}^{m_{k}}\right)$, we let

$$
\mu_{\lambda}=1^{m_{1}} m_{1} ! 2^{m_{2}} m_{2} ! \cdots k^{m_{k}} m_{k} !
$$

Theorem 1.2 For positive integers $p, r, q$ with $q \geq 2$, we have

$$
\begin{gathered}
\sum_{|\alpha|=n+p+r+q} \sum_{1 \leq \ell_{1}<\ell_{2}<\cdots<\ell_{p}}\left(\begin{array}{c}
\alpha_{p+r} \\
q-1
\end{array}\right) \prod_{i=1}^{p} \ell_{i}^{-\alpha_{i}}\left[\prod_{j=1}^{r-1}\left(\ell_{p}+j\right)^{-\alpha_{p+j}}\right]\left(\ell_{p}+r\right)^{-\alpha_{p+r}-1} \\
\sum_{1 \leq k_{1}<k_{2}<\cdots<k_{q}} \frac{1}{k_{1} \cdots k_{q}\left(k_{q}+1\right) \cdots\left(k_{q}+r-1\right)\left(k_{q}+r\right)^{p+1}} \\
\times \sum_{\lambda \vdash n} \mu_{\lambda}^{-1} h_{\lambda_{1}} h_{\lambda_{2}} \cdots h_{\lambda_{g}},
\end{gathered}
$$

where the summation ranges over all partitions $\lambda=\left(\lambda_{1}, \lambda_{2}, \ldots, \lambda_{g}\right)$ of $n$ and $h_{m}=\sum_{k=k_{1}}^{k_{q}+r} \frac{1}{k^{m}}$.

We investigate the Euler sum with two branches $G_{n}(p, q)$ (see (8) below for a definition) and drive important properties in Section 4. In Section 5, we consider the deformed iterated integral with a real parameter $a>-1$,

$$
F_{r, q}(a)=\int_{E_{r+q+2}}\left(\frac{t_{1}}{t_{r+q+2}}\right)^{a}\left(\prod_{i=1}^{r} \frac{d t_{i}}{1-t_{i}}\right)\left(\prod_{k=r+1}^{r+q+1} \frac{d t_{k}}{t_{k}}\right) d t_{r+q+2},
$$

and prove the following.

Theorem 1.3 Suppose that $n, p, q$ are nonnegative integers with $p \geq 1$. Then

$$
\begin{aligned}
& \sum_{|\alpha|=p+q+n} \sum_{1 \leq \ell_{1}<\ell_{2}<\cdots<\ell_{p}}\left(\begin{array}{c}
\alpha_{p} \\
q+1
\end{array}\right) \ell_{1}^{-\alpha_{1}} \cdots \ell_{p-1}^{-\alpha_{p-1}} \ell_{p}^{-\alpha_{p}-1}\left(\ell_{p}+1\right)^{-1} \\
= & \sum_{j=0}^{q} \sum_{k=0}^{n}(-1)^{j+k} G_{p+1}(q-j, n-k)+\sum_{k=0}^{n}(-1)^{k+q+1}+\sum_{k=0}^{n-1}(-1)^{n-1-k} \beta_{k}
\end{aligned}
$$

with

$$
\beta_{m}=\sum_{1 \leq \ell_{1}<\ell_{2}<\cdots<\ell_{q+1}} \frac{1}{\ell_{1}\left(\ell_{1}+1\right)\left(\ell_{2}+1\right) \cdots\left(\ell_{q}+1\right)\left(\ell_{q+1}+1\right)^{p+1}} \sum_{\lambda \vdash m} \mu_{\lambda}^{-1} H_{\lambda_{1}} H_{\lambda_{2}} \cdots H_{\lambda_{g}},
$$

where

$$
H_{j}=\sum_{\ell=\ell_{1}}^{\ell_{q+1}+1} \frac{1}{\ell^{j}}
$$


A corollary of Theorem 1.3 (when taking $q=0$ into (3)) is the weighted sum formula first obtained in (Arakawa \& Kaneko, 1999; Ohno, 1999):

$$
\sum_{|\alpha|=n+p} \alpha_{p} \zeta\left(\alpha_{1}, \ldots, \alpha_{p-1}, \alpha_{p}+1\right)=\zeta^{\star}\left(\{1\}^{n}, p+1\right)
$$

\section{Some Analogues of the Sum Formula}

Here is an analogue of the sum formula obtained in the similar way by considering different iterated integrals with additional factors.

Theorem 2.1 Suppose that $p$ is a positive integer and

$$
P_{1}(x)=\prod_{j=1}^{r}(x+j), \quad P_{2}(x)=x P_{1}(x) .
$$

Then for any positive integers $r$ and $n$, we have

$$
\begin{aligned}
& \sum_{|\alpha|=p+r-1+n} \sum_{1 \leq \ell_{1}<\ell_{2}<\cdots<\ell_{p}} \prod_{i=1}^{p} \ell_{i}^{-\alpha_{i}} \prod_{j=1}^{r-1}\left(\ell_{p}+j\right)^{-\alpha_{p+j}}\left(\ell_{p}+r\right)^{-1} \\
= & \frac{1}{r^{p}} \sum_{j=1}^{r} \frac{1}{j^{n+1} P_{1}^{\prime}(-j)}-\sum_{k=1}^{\infty} \frac{1}{(k+r)^{p}} \sum_{j=0}^{r} \frac{1}{(k+j)^{n} P_{2}^{\prime}(-j)} .
\end{aligned}
$$

proof. For real $a>-1$, consider the iterated integral given by (1) with $q=0$. That is,

$$
I_{p, r, 0}(a)=\int_{E_{p+r}}\left(\frac{t_{1}}{t_{p+r}}\right)^{a}\left(\prod_{i=1}^{p} \frac{d t_{i}}{1-t_{i}}\right)\left(\prod_{j=p+1}^{p+r} d t_{j}\right), \quad r \geq 1 .
$$

It can be evaluated as

$$
\sum_{1 \leq \ell_{1}<\ell_{2}<\cdots<\ell_{p}} \prod_{i=1}^{p}\left(\ell_{i}+a\right)^{-1} \prod_{j=1}^{r-1}\left(\ell_{p}+j+a\right)^{-1}\left(\ell_{p}+r\right)^{-1} .
$$

Also its dual is given by

$$
\int_{E_{r+p}}\left(\frac{1-u_{r+p}}{1-u_{1}}\right)^{a}\left(\prod_{j=1}^{r} d u_{j}\right)\left(\prod_{i=r+1}^{r+p} \frac{d u_{i}}{u_{i}}\right) .
$$

Just rewrite $d u_{1}$ as $d u_{1} /\left(1-u_{1}\right)-u_{1} d u_{1} /\left(1-u_{1}\right)$ and note that

$$
\int_{0}^{1} u^{x-1}(1-u)^{y-1} d u=\frac{\Gamma(x) \Gamma(y)}{\Gamma(x+y)}
$$

so the dual can be evaluated as the difference

$$
\begin{gathered}
\sum_{k=1}^{\infty} \frac{1}{k(k+1) \cdots(k+r-2)(k+r-1)^{p+1}}\left[\frac{\Gamma(k+a) \Gamma(k+r)}{\Gamma(k) \Gamma(k+r+a)}\right] \\
-\sum_{k=1}^{\infty} \frac{1}{(k+1)(k+2) \cdots(k+r-1)(k+r)^{p+1}}\left[\frac{\Gamma(k+a) \Gamma(k+r+1)}{\Gamma(k) \Gamma(k+r+1+a)}\right],
\end{gathered}
$$

or with a term by term cancellation

$$
\frac{1}{r^{p}(a+1)(a+2) \cdots(a+r)}+\sum_{k=1}^{\infty} \frac{a}{(k+r)^{p}(k+a)(k+1+a) \cdots(k+r+a)} .
$$

Note that

$$
\frac{1}{(a+1)(a+2) \cdots(a+r)}=\sum_{j=1}^{r} \frac{1}{(a+j) P_{1}^{\prime}(-j)}
$$


and

$$
\frac{1}{(k+a)(k+1+a) \cdots(k+r+a)}=\sum_{j=0}^{r} \frac{1}{(k+j+a) P_{2}^{\prime}(-j)}
$$

so that

$$
\left.\frac{(-1)^{n-1}}{(n-1) !}\left(\frac{d^{n-1}}{d a^{n-1}}\right) \frac{1}{(k+a)(k+1+a) \cdots(k+r+a)}\right|_{a=0}=\sum_{j=0}^{r} \frac{1}{(k+j)^{n} P_{2}^{\prime}(-j)} .
$$

This proves our assertion.

Here we exhibit the cases $r=1,2$ and 3, respectively. For a positive integer $p$ and a nonnegative integer $n$, we have

(i)

$$
\sum_{|\alpha|=n+p} \sum_{1 \leq \ell_{1}<\ell_{2}<\cdots<\ell_{p}} \ell_{1}^{-\alpha_{1}} \ell_{2}^{-\alpha_{2}} \cdots \ell_{p}^{-\alpha_{p}}\left(\ell_{p}+1\right)^{-1}=\zeta(n+p)-\sum_{k=1}^{\infty} \frac{1}{k^{n}(k+1)^{p}} .
$$

(ii)

$$
\begin{aligned}
& \sum_{|\alpha|=n+p+1} \sum_{1 \leq \ell_{1}<\ell_{2}<\cdots<\ell_{p}} \ell_{1}^{-\alpha_{1}} \ell_{2}^{-\alpha_{2}} \cdots \ell_{p}^{-\alpha_{p}}\left(\ell_{p}+1\right)^{-\alpha_{p+1}}\left(\ell_{p}+2\right)^{-1} \\
= & \sum_{k=1}^{\infty} \frac{1}{k^{n}(k+1)^{p}}-\frac{1}{2} \sum_{k=1}^{\infty} \frac{1}{k^{n}(k+2)^{p}}-\frac{1}{2} \zeta(n+p)+\frac{1}{2} \quad(p \geq 2) .
\end{aligned}
$$

(iii)

$$
\begin{aligned}
& \sum_{|\alpha|=n+p+2} \sum_{1 \leq \ell_{1}<\ell_{2}<\cdots<\ell_{p}} \prod_{j=1}^{p} \ell_{j}^{-\alpha_{j}}\left(\ell_{p}+1\right)^{-\alpha_{p+1}}\left(\ell_{p}+2\right)^{-\alpha_{p+2}}\left(\ell_{p}+3\right)^{-1} \\
= & \frac{1}{6} \zeta(n+p)-\frac{1}{2} \sum_{k=1}^{\infty} \frac{1}{k^{n}(k+1)^{p}}+\frac{1}{2} \sum_{k=1}^{\infty} \frac{1}{k^{n}(k+2)^{p}} \\
& -\frac{1}{6} \sum_{k=1}^{\infty} \frac{1}{k^{n}(k+3)^{p}}+\frac{1}{2^{p+1}}-\frac{1}{6}\left(1+\frac{1}{2^{n+1}}\right) \quad(p \geq 2) .
\end{aligned}
$$

\section{A Generalization of the Sum Formula}

Recall the iterated integral given by (1). Theorem 2.1 deals with the case $q=0$. For the case $q=1$, we can even study more general form. Let $b$ be a nonnegative integer. In our next consideration, we begin with the double-attached iterated integral

$$
\int_{E_{p+r+1}}\left(\frac{t_{1}}{t_{p+r+1}}\right)^{a}\left(\prod_{i=1}^{p} \frac{d t_{i}}{1-t_{i}}\right)\left(\prod_{j=p+1}^{p+r} d t_{j}\right) \frac{\left(1-t_{p+r+1}\right)^{b} d t_{p+r+1}}{t_{p+r+1}} .
$$

Note that the above is the integral $I_{p, r, 1}(a)$ with an additional attached factor $\left(1-t_{p+r+1}\right)^{b}$ and it can be evaluated as

$$
\sum_{1 \leq \ell_{1}<\ell_{2}<\cdots<\ell_{p}} b ! \prod_{i=1}^{p}\left(\ell_{i}+a\right)^{-1} \prod_{j=1}^{r-1}\left(\ell_{p}+a+j\right)^{-1} \prod_{m=0}^{b}\left(\ell_{p}+r+m\right)^{-1} .
$$

From this, we obtain the contribution of the additional attached factor $\left(1-t_{p+r+1}\right)^{b}$ corresponds to the factor $b$ ! and the increment of the product $\prod_{m=0}^{b}\left(\ell_{p}+r+m\right)^{-1}$ after the evaluation. Also notice that the term $\prod_{j=1}^{r-1}\left(\ell_{p}+a+j\right)^{-1}$ vanishes when $r \leq 1$.

In the following, we need the differentiation of quotients of gamma functions

$$
\theta_{n}=\left.\frac{(-1)^{n}}{n !}\left(\frac{d^{n}}{d x^{n}}\right) \frac{\Gamma\left(k_{1}+x\right) \Gamma\left(k_{2}\right)}{\Gamma\left(k_{1}\right) \Gamma\left(k_{2}+x\right)}\right|_{x=0}
$$


with positive integers $k_{1}<k_{2}$. Let

$$
g(x)=\frac{\Gamma\left(k_{1}+x\right) \Gamma\left(k_{2}\right)}{\Gamma\left(k_{1}\right) \Gamma\left(k_{2}+x\right)}
$$

and $\psi(x)$ be the digamma function defined by

$$
\psi(x)=\frac{d}{d x} \log \Gamma(x)=\frac{\Gamma^{\prime}(x)}{\Gamma(x)} .
$$

It is easy to see that

$$
g^{\prime}(x)=-g(x)\left[\psi\left(k_{2}+x\right)-\psi\left(k_{1}+x\right)\right]=-g(x) h_{1}(x)
$$

with

$$
h_{m}(x)=\sum_{\ell=k_{1}}^{k_{2}-1} \frac{1}{(\ell+x)^{m}} .
$$

So that

$$
n \theta_{n}=\sum_{j=0}^{n-1} \theta_{j} h_{n-j}(0)
$$

We also need the following result from combinatorics (MacDonald, 1995; Stanley, 1999).

Proposition 3.1 For two fixed positive integers $k_{1}, k_{2}$ with $k_{1}<k_{2}$, we have

$$
\theta_{n}=\left.\frac{(-1)^{n}}{n !}\left(\frac{d^{n}}{d x^{n}}\right) \frac{\Gamma\left(k_{1}+x\right) \Gamma\left(k_{2}\right)}{\Gamma\left(k_{1}\right) \Gamma\left(k_{2}+x\right)}\right|_{x=0}=\sum_{\lambda \vdash n} \mu_{\lambda}^{-1} L_{\lambda_{1}} L_{\lambda_{2}} \cdots L_{\lambda_{g}}
$$

where $L_{m}=\sum_{\ell=k_{1}}^{k_{2}-1} \frac{1}{\ell^{m}}$.

Theorem 3.2 Let $p$ be a positive integer and $a>-1$ be a real. For nonnegative integers $b, n$ and $r$, we have

$$
\begin{aligned}
& \sum_{|\alpha|=p+r+n} \sum_{1 \leq \ell_{1}<\ell_{2}<\cdots<\ell_{p}} b ! \prod_{i=1}^{p} \ell_{i}^{-\alpha_{i}} \prod_{j=1}^{r-1}\left(\ell_{p}+j\right)^{-\alpha_{p+j}} \prod_{m=0}^{b}\left(\ell_{p}+r+m\right)^{-1} \\
= & \sum_{k=1}^{\infty} \frac{1}{(k+b)(k+b+1) \cdots(k+b+r-1)(k+b+r)^{p+1}} \sum_{\lambda \vdash n} \mu_{\lambda}^{-1} v_{\lambda_{1}} \cdots v_{\lambda_{g}},
\end{aligned}
$$

here

$$
v_{m}=\sum_{\ell=0}^{r+b} \frac{1}{(k+\ell)^{m}} .
$$

proof. For real number $a>-1$, the dual of the double-attached iterated integral (5) is given by

$$
\int_{E_{r+p+1}}\left(\frac{1-u_{r+p+1}}{1-u_{1}}\right)^{a} \frac{u_{1}^{b} d u_{1}}{1-u_{1}}\left(\prod_{j=2}^{r+1} d u_{j}\right)\left(\prod_{i=r+2}^{r+p+1} \frac{d u_{i}}{u_{i}}\right)
$$

and it can be evaluated as

$$
\begin{aligned}
& \sum_{k=1}^{\infty} \frac{1}{(k+b)(k+b+1) \cdots(k+b+r-1)(k+b+r)^{p+1}} \\
& \times \frac{\Gamma(k+a) \Gamma(k+r+b+1)}{\Gamma(k) \Gamma(k+r+b+a+1)} .
\end{aligned}
$$

Our assertion then follows from the identity

$$
\begin{aligned}
\sum_{1 \leq \ell_{1}<\ell_{2}<\cdots<\ell_{p}} b ! \prod_{i=1}^{p}\left(\ell_{i}+a\right)^{-1} \prod_{j=1}^{r-1}\left(\ell_{p}+a+j\right)^{-1} \prod_{m=0}^{b}\left(\ell_{p}+r+m\right)^{-1} \\
=\sum_{k=1}^{\infty} \frac{1}{(k+b)(k+b+1) \cdots(k+b+r-1)(k+b+r)^{p+1}} \\
\times \frac{\Gamma(k+a) \Gamma(k+r+b+1)}{\Gamma(k) \Gamma(k+r+b+a+1)}
\end{aligned}
$$


after applying the operator $\left.\frac{(-1)^{n}}{n !}\left(\frac{d^{n}}{d a^{n}}\right)\right|_{a=0}$ and Proposition 3.1.

When $b=r=0$ in Theorem 3.2, we get the well-known sum formula of multiple zeta values.

Corollary 3.3 [Sum Formula (Granville, 1997)] For a nonnegative integer $n$, we have

$$
\begin{aligned}
& \sum_{|\alpha|=n+p} \sum_{1 \leq \ell_{1}<\ell_{2}<\cdots<\ell_{p}} \ell_{1}^{-\alpha_{1}} \cdots \ell_{p-1}^{-\alpha_{p-1}} \ell_{p}^{-\alpha_{p}-1} \\
= & \sum_{|\alpha|=n+p} \zeta\left(\alpha_{1}, \alpha_{2}, \ldots, \alpha_{p}+1\right)=\zeta(n+p+1) .
\end{aligned}
$$

Some other special cases of (6) are also of special interesting. For any nonnegative integer $n$, we have

(i) $(b=0, r=1)$

$$
\begin{aligned}
& \sum_{|\alpha|=n+p+1} \sum_{1 \leq \ell_{1}<\ell_{2}<\cdots<\ell_{p}} \ell_{1}^{-\alpha_{1}} \ell_{2}^{-\alpha_{2}} \cdots \ell_{p}^{-\alpha_{p}}\left(\ell_{p}+1\right)^{-\alpha_{p+1}-1} \\
= & \sum_{k=1}^{\infty} \frac{1}{k^{n+1}(k+1)^{p}}-\zeta(n+p+1)+1
\end{aligned}
$$

(ii) $(b=1, r=0)$

$$
\begin{aligned}
& \sum_{|\alpha|=n+p} \sum_{1 \leq \ell_{1}<\ell_{2}<\cdots<\ell_{p}} \ell_{1}^{-\alpha_{1}} \ell_{2}^{-\alpha_{2}} \cdots \ell_{p}^{-\alpha_{p}}\left[\ell_{p}\left(\ell_{p}+1\right)\right]^{-1} \\
= & \zeta(n+p+1)-\zeta(n+p)+\sum_{k=1}^{\infty} \frac{1}{k^{n}(k+1)^{p}} \quad \text { for } p \geq 2 ;
\end{aligned}
$$

(iii) $(b=2, r=0)$

$$
\begin{aligned}
& \sum_{|\alpha|=n+p} \sum_{1 \leq \ell_{1}<\ell_{2}<\cdots<\ell_{p}} \ell_{1}^{-\alpha_{1}} \ell_{2}^{-\alpha_{2}} \cdots \ell_{p}^{-\alpha_{p}}\left[\ell_{p}\left(\ell_{p}+1\right)\left(\ell_{p}+2\right)\right]^{-1} \\
= & \frac{1}{4}\left[\zeta(n+p-1)-3 \zeta(n+p)+2 \zeta(n+p+1)+\sum_{k=1}^{\infty} \frac{k+1}{k^{n}(k+2)^{p}}-\sum_{k=1}^{\infty} \frac{2(k-1)}{k^{n}(k+1)^{p}}\right],
\end{aligned}
$$

for $p \geq 3$.

The general case $q \geq 2$ of the evaluation of the integral (1) in terms of infinite sum is given by

$$
\sum_{1 \leq \ell_{1}<\ell_{2}<\cdots<\ell_{p}} \prod_{i=1}^{p}\left(\ell_{i}+a\right)^{-1} \prod_{j=1}^{r-1}\left(\ell_{p}+a+j\right)^{-1}\left(\ell_{p}+a+r\right)^{-q}\left(\ell_{p}+r\right)^{-1} .
$$

On the other hand, the dual of (1) is given by

$$
I_{p, r, q}^{\vee}(a)=\int_{E_{q+r+p}}\left(\frac{1-u_{q+r+p}}{1-u_{1}}\right)^{a}\left(\prod_{i=1}^{q} \frac{d u_{i}}{1-u_{i}}\right)\left(\prod_{j=q+1}^{q+r} d u_{j}\right)\left(\prod_{k=q+r+1}^{q+r+p} \frac{d u_{k}}{u_{k}}\right),
$$

and which is, in terms of infinite sum,

$$
\sum_{1 \leq k_{1}<k_{2}<\cdots<k_{q}} \frac{1}{k_{1} \cdots k_{q}\left(k_{q}+1\right) \cdots\left(k_{q}+r-1\right)\left(k_{q}+r\right)^{p+1}} \cdot \frac{\Gamma\left(k_{1}+a\right) \Gamma\left(k_{q}+r+1\right)}{\Gamma\left(k_{1}\right) \Gamma\left(k_{q}+r+a+1\right)} .
$$

Proof of Theorem 1.2. From the above discussion we simply obtain the identity

$$
\begin{aligned}
& \sum_{1 \leq \ell_{1}<\ell_{2}<\cdots<\ell_{p}}\left[\prod_{i=1}^{p}\left(\ell_{i}+a\right) \prod_{j=1}^{r-1}\left(\ell_{p}+a+j\right)\left(\ell_{p}+a+r\right)^{q}\left(\ell_{p}+r\right)\right]^{-1} \\
&=\sum_{1 \leq k_{1}<k_{2}<\cdots<k_{q}} \frac{1}{k_{1} \cdots k_{q}\left(k_{q}+1\right) \cdots\left(k_{q}+r-1\right)\left(k_{q}+r\right)^{p+1}} \\
& \times \frac{\Gamma\left(k_{1}+a\right) \Gamma\left(k_{q}+r+1\right)}{\Gamma\left(k_{1}\right) \Gamma\left(k_{q}+r+a+1\right)} .
\end{aligned}
$$


The assertion follows by applying the operator $\left.\frac{(-1)^{n}}{n !}\left(\frac{d^{n}}{d a^{n}}\right)\right|_{a=0}$ on the both sides of the identity (7) and a simple differential result as follows

$$
\begin{aligned}
& \left.\frac{(-1)^{n}}{n !}\left[\frac{1}{\left(\ell_{1}+a\right)\left(\ell_{2}+a\right) \cdots\left(\ell_{p-1}+a\right)\left(\ell_{p}+a\right)^{m+2}}\right]^{(n)}\right|_{a=0} \\
= & \sum_{|\alpha|=p+m+n}\left(\begin{array}{c}
\alpha_{p} \\
m+1
\end{array}\right) \frac{1}{\ell_{1}^{\alpha_{1}} \ell_{2}^{\alpha_{2}} \cdots \ell_{p-1}^{\alpha_{p-1}} \ell_{p}^{\alpha_{p}+1}},
\end{aligned}
$$

for $m, n \geq 0$.

\section{Euler Sums With Two Branches}

For three nonnegative integers $n, p, q$ with $n \geq 2$, we define the Euler sum with two branches $G_{n}(p, q)$ (Eie, Liaw \& Ong, 2009) by

$$
G_{n}(p, q)=\sum_{1 \leq k_{1}<k_{2}<\cdots<k_{p+1}} \frac{1}{k_{1} k_{2} \cdots k_{p} k_{p+1}^{n}} \sum_{1 \leq \ell_{1} \leq \cdots \leq \ell_{q} \leq k_{p+1}} \frac{1}{\ell_{1} \ell_{2} \cdots \ell_{q}} .
$$

According to (p.246, Eie, 2009), it has the simple integral representation

$$
\frac{1}{p ! q !(n-2) !} \int_{E_{2}}\left(\log \frac{1}{1-t_{1}}\right)^{p}\left(\log \frac{1}{1-t_{2}}\right)^{q}\left(\log \frac{t_{2}}{t_{1}}\right)^{n-2} \frac{d t_{1} d t_{2}}{\left(1-t_{1}\right) t_{2}}
$$

and can be decomposed into usual multiple zeta values as (Proposition 5, Eie, Liaw, \& Ong, 2009)

$$
G_{n}(p, q)=\sum_{k=p+1}^{p+q+1}\left(\begin{array}{c}
k-1 \\
p
\end{array}\right) \sum_{|\alpha|=p+q+1} \zeta\left(\alpha_{1}, \ldots, \alpha_{k-1}, \alpha_{k}+n-1\right) .
$$

We note that (9) and (10) together imply the well-known restricted sum formula (Eie, Liaw, \& Ong, 2009),

$$
\sum_{|\alpha|=m+n+1} \zeta\left(\{1\}^{p}, \alpha_{1}, \ldots, \alpha_{m}, \alpha_{m+1}+1\right)=\sum_{|c|=p+m+1} \zeta\left(c_{1}, \ldots, c_{p}, c_{p+1}+n+1\right),
$$

for nonnegative integers $m, n$ and $p$.

It is quite surprising that $G_{2 n}(p, q)$ comes from the differentiation of some multiple zeta values with parameters.

Proposition 4.1 Suppose that $p, q$ are nonnegative integers and

$$
H_{p, q}(a)=\sum_{1 \leq \ell_{1}<\ell_{2}<\cdots<\ell_{q+1}} \frac{1}{\ell_{1} \cdots \ell_{q} \ell_{q+1}^{p+1}}\left[\frac{\Gamma(1+a) \Gamma\left(\ell_{q+1}+1\right)}{\Gamma\left(\ell_{q+1}+1+a\right)}\right] .
$$

Then for nonnegative integer $n$

$$
\left.\frac{(-1)^{n}}{n !} H_{p, q}^{(n)}(a)\right|_{a=0}=G_{p+1}(q, n)
$$

proof. Let $w=p+q+1$. The multiple zeta value $H_{p, q}(a)$ can be expressed as the iterated integral

$$
\int_{E_{p+q+1}}\left(\prod_{j=1}^{q+1} \frac{d u_{j}}{1-u_{j}}\right)\left(\prod_{k=q+2}^{p+q} \frac{d u_{k}}{u_{k}}\right) \frac{\left(1-u_{p+q+1}\right)^{a} d u_{p+q+1}}{u_{p+q+1}} .
$$

So that

$$
\left.\frac{(-1)^{n}}{n !} H_{p, q}^{(n)}(a)\right|_{a=0}=\frac{1}{n !} \int_{E_{p+q+1}}\left(\log \frac{1}{1-u_{p+q+1}}\right)^{n}\left(\prod_{j=1}^{q+1} \frac{d u_{j}}{1-u_{j}}\right)\left(\prod_{k=q+2}^{p+q+1} \frac{d u_{k}}{u_{k}}\right) .
$$

Fix $u_{q+1}$ and $u_{p+q+1}$ as new dummy variables $t_{1}$ and $t_{2}$ and then integrate with respect to the rest of variables, the above iterated integral becomes

$$
\frac{1}{q ! n !(p-1) !} \int_{E_{2}}\left(\log \frac{1}{1-t_{1}}\right)^{q}\left(\log \frac{1}{1-t_{2}}\right)^{n}\left(\log \frac{t_{2}}{t_{1}}\right)^{p-1} \frac{d t_{1} d t_{2}}{\left(1-t_{1}\right) t_{2}}
$$


which is precisely the double integral representation of $G_{p+1}(q, n)$ by (8).

In light of Proposition 3.1, we obtain another expression of $G_{p+1}(q, n)$.

Proposition 4.2 For integers $p, q, n$ with $p \geq 1$ and $q, n \geq 0$, we have

$$
G_{p+1}(q, n)=\sum_{1 \leq \ell_{1}<\ell_{2}<\cdots<\ell_{q+1}} \frac{1}{\ell_{1} \cdots \ell_{q} \ell_{q+1}^{p+1}} \sum_{\lambda \vdash n} \mu_{\lambda}^{-1} H_{\lambda_{1}}^{\prime} H_{\lambda_{2}}^{\prime} \cdots H_{\lambda_{g}}^{\prime}
$$

with $H_{m}^{\prime}=\sum_{k=1}^{\ell_{q+1}} \frac{1}{k^{m}}$.

\section{Deformation of Multiple Zeta Values}

Throughout this section, fix $w=p+q+1$. Recall the deformed iterated integral $F_{r, q}(a)$ defined by (2). Simply its dual is given by

$$
F_{r, q}^{\vee}(a)=\int_{E_{q+r+2}}\left(\frac{1-u_{q+r+2}}{1-u_{1}}\right)^{a} d u_{1}\left(\prod_{i=2}^{q+2} \frac{d u_{i}}{1-u_{i}}\right)\left(\prod_{k=q+3}^{q+r+2} \frac{d u_{k}}{u_{k}}\right) .
$$

In addition, we need a representation of the alternating sum of $H_{p, j}(a)$ with $0 \leq j \leq q$.

Proposition 5.1 For a pair of integers $p, q$ with $p \geq 1, q \geq 0$, let

$$
\widetilde{H}_{p, q}(a)=\sum_{1<\ell_{1}<\ell_{2}<\cdots<\ell_{q+1}} \frac{1}{\ell_{1} \cdots \ell_{q} \ell_{q+1}^{p+1}}\left[\frac{\Gamma(1+a) \Gamma\left(\ell_{q+1}+1\right)}{\Gamma\left(\ell_{q+1}+1+a\right)}\right] .
$$

Then

$$
\widetilde{H}_{p, q}(a)=\sum_{j=0}^{q}(-1)^{j} H_{p, q-j}(a)+(-1)^{q+1} \frac{1}{1+a}
$$

and

$$
\left.\frac{(-1)^{n}}{n !} \widetilde{H}_{p, q}^{(n)}(a)\right|_{a=0}=\sum_{j=0}^{q}(-1)^{q-j} G_{p+1}(j, n)+(-1)^{q+1} .
$$

proof. The first assertion follows from

$$
\widetilde{H}_{p, q}(a)=H_{p, q}(a)-\widetilde{H}_{p, q-1}(a)
$$

and

$$
\widetilde{H}_{p, 0}(a)=H_{p, 0}(a)-\frac{1}{1+a} .
$$

The second assertion follows from Proposition 4.1.

On the other hand, let $K_{p, q}(a)$ be the multiple zeta value with a parameter $a$ defined by

$$
\begin{aligned}
K_{p, q}(a) & \sum_{1 \leq \ell_{1}<\ell_{2}<\cdots<\ell_{q+1}} \frac{1}{\ell_{1}\left(\ell_{1}+1\right)\left(\ell_{2}+1\right) \cdots\left(\ell_{q}+1\right)\left(\ell_{q+1}+1\right)^{p+1}}\left[\frac{\Gamma\left(\ell_{1}+a\right) \Gamma\left(\ell_{q+1}+2\right)}{\Gamma\left(\ell_{1}\right) \Gamma\left(\ell_{q+1}+2+a\right)}\right]
\end{aligned}
$$

which comes from the evaluation of the iterated integral

$$
\int_{E_{w+1}}\left(\frac{1-u_{w+1}}{1-u_{1}}\right)^{a} \frac{d u_{1}}{1-u_{1}} d u_{2}\left(\prod_{j=3}^{q+2} \frac{d u_{j}}{1-u_{j}}\right)\left(\prod_{k=q+3}^{w+1} \frac{d u_{k}}{u_{k}}\right) .
$$

However, for $q \geq 1$, if we rewrite $K_{p, q}(a)$ as the difference

$$
\begin{aligned}
\sum_{1 \leq \ell_{1}<\ell_{2}<\cdots<\ell_{q+1}} & {\left[\frac{1}{\ell_{1}\left(\ell_{2}+1\right) \cdots\left(\ell_{q}+1\right)\left(\ell_{q+1}+1\right)^{p+1}}\right.} \\
& \left.-\frac{1}{\left(\ell_{1}+1\right)\left(\ell_{2}+1\right) \cdots\left(\ell_{q}+1\right)\left(\ell_{q+1}+1\right)^{p+1}}\right]\left[\frac{\Gamma\left(\ell_{1}+a\right) \Gamma\left(\ell_{q+1}+2\right)}{\Gamma\left(\ell_{1}\right) \Gamma\left(\ell_{q+1}+2+a\right)}\right] .
\end{aligned}
$$


Then it is equal to the evaluation of the difference of two integrals

$$
\begin{aligned}
& \int_{E_{w}}\left(\frac{1-u_{w}}{1-u_{1}}\right)^{a} \frac{d u_{1}}{1-u_{1}} \frac{u_{2} d u_{2}}{1-u_{2}}\left(\prod_{j=3}^{q+1} \frac{d u_{j}}{1-u_{j}}\right)\left(\prod_{k=q+2}^{w} \frac{d u_{k}}{u_{k}}\right) \\
& -\int_{E_{w}}\left(\frac{1-u_{w}}{1-u_{1}}\right)^{a} \frac{u_{1} d u_{1}}{1-u_{1}} \frac{d u_{2}}{1-u_{2}}\left(\prod_{j=3}^{q+1} \frac{d u_{j}}{1-u_{j}}\right)\left(\prod_{k=q+2}^{w} \frac{d u_{k}}{u_{k}}\right),
\end{aligned}
$$

or

$$
\begin{aligned}
& -\int_{E_{w}}\left(\frac{1-u_{w}}{1-u_{1}}\right)^{a} \frac{d u_{1}}{1-u_{1}} d u_{2}\left(\prod_{j=3}^{q+1} \frac{d u_{j}}{1-u_{j}}\right)\left(\prod_{k=q+2}^{w} \frac{d u_{k}}{u_{k}}\right) \\
& +\int_{E_{w}}\left(\frac{1-u_{w}}{1-u_{1}}\right)^{a} d u_{1} \frac{d u_{2}}{1-u_{2}}\left(\prod_{j=3}^{q+1} \frac{d u_{j}}{1-u_{j}}\right)\left(\prod_{k=q+2}^{w} \frac{d u_{k}}{u_{k}}\right)
\end{aligned}
$$

which is equal to $-K_{p, q-1}(a)+F_{p, q-1}^{\vee}(a)$. So that we have proved the following.

Proposition 5.2 For a pair of positive integers $p, q$ and real number $a>-1$, let $K_{p, q}(a)$ be defined as (13). Then

$$
K_{p, q}(a)=F_{p, q-1}^{\vee}(a)-K_{p, q-1}(a)
$$

and

$$
K_{p, q}(a)=\sum_{j=0}^{q-1}(-1)^{q-1-j} F_{p, j}^{\vee}(a)+(-1)^{q} K_{p, 0}(a) .
$$

Proposition 5.3 Notations as shown in (11), (12) and (13). Then for real number $a>-1$ and $a \neq 1$, we have

$$
F_{p, q}^{\vee}(a)=\frac{1}{1-a} \widetilde{H}_{p, q}(a)-\frac{a}{1-a} K_{p, q}(a) .
$$

proof. The dual can be expressed as the difference

$$
\begin{aligned}
& \int_{E_{w+1}}\left(\frac{1-u_{w+1}}{1-u_{1}}\right)^{a} \frac{d u_{1}}{1-u_{1}}\left(\prod_{j=2}^{q+2} \frac{d u_{j}}{1-u_{j}}\right)\left(\prod_{k=q+3}^{w+1} \frac{d u_{k}}{u_{k}}\right) \\
& -\int_{E_{w+1}}\left(\frac{1-u_{w+1}}{1-u_{1}}\right)^{a} \frac{u_{1} d u_{1}}{1-u_{1}}\left(\prod_{j=2}^{q+2} \frac{d u_{j}}{1-u_{j}}\right)\left(\prod_{k=q+3}^{w+1} \frac{d u_{k}}{u_{k}}\right),
\end{aligned}
$$

and hence can be evaluated as

$$
\begin{aligned}
& \sum_{1 \leq \ell_{1}<\ell_{2}<\cdots<\ell_{q+2}} \frac{1}{\ell_{1} \cdots \ell_{q+1} \ell_{q+2}^{p+1}}\left[\frac{\Gamma\left(\ell_{1}+a\right) \Gamma\left(\ell_{q+2}+1\right)}{\Gamma\left(\ell_{1}\right) \Gamma\left(\ell_{q+2}+1+a\right)}\right] \\
& -\sum_{1 \leq \ell_{1}<\ell_{2}<\cdots<\ell_{q+2}} \frac{1}{\left(\ell_{1}+1\right) \cdots\left(\ell_{q+1}+1\right)\left(\ell_{q+2}+1\right)^{p+1}}\left[\frac{\Gamma\left(\ell_{1}+a\right) \Gamma\left(\ell_{q+2}+2\right)}{\Gamma\left(\ell_{1}\right) \Gamma\left(\ell_{q+2}+2+a\right)}\right] .
\end{aligned}
$$

According to $\ell_{1}=1$ or $\ell_{2}>1$, the first series is separated into two sum

$$
\widetilde{H}_{p, q}(a)+\sum_{1 \leq \ell_{1}<\ell_{2}<\cdots<\ell_{q+2}} \frac{1}{\left(\ell_{1}+1\right) \cdots\left(\ell_{q+1}+1\right)\left(\ell_{q+2}+1\right)^{p+1}}\left[\frac{\Gamma\left(\ell_{1}+1+a\right) \Gamma\left(\ell_{q+2}+2\right)}{\Gamma\left(\ell_{1}+1\right) \Gamma\left(\ell_{q+2}+2+a\right)}\right] .
$$

So after a term by term cancellation between the above series and the negative part of $F_{p, q}^{\vee}(a)$ in (15) in light of the functional equation

we conclude that

$$
\frac{\Gamma\left(\ell_{1}+1+a\right)}{\Gamma\left(\ell_{1}+1\right)}=\frac{\left(\ell_{1}+a\right) \Gamma\left(\ell_{1}+a\right)}{\ell_{1} \Gamma\left(\ell_{1}\right)},
$$

$$
F_{p, q}^{\vee}(a)=\widetilde{H}_{p, q}(a)+a K_{p, q+1}(a) .
$$


Our assertion then follows by (14).

We are now at the stage of giving a proof of Theorem 1.3.

Proof of Theorem 1.3. Suppose that

$$
\gamma_{m}=\left.\frac{(-1)^{m}}{m !} \widetilde{H}_{p, q}^{(m)}(a)\right|_{a=0}
$$

The relation

$$
F_{p, q}^{\vee}(a)=\frac{1}{1-a} \widetilde{H}_{p, q}(a)-\frac{a}{1-a} K_{p, q}(a)
$$

then implies that the sum on the left side (3) is equal to

$$
\sum_{k=0}^{n}(-1)^{k} \gamma_{n-k}+\sum_{k=0}^{n-1}(-1)^{n-1-k} \beta_{k}
$$

Now it suffices to evaluate $\gamma_{n-k}$ in more explicit form. By Proposition 4.1 and 5.1, we have

$$
\sum_{k=0}^{n}(-1)^{k} \gamma_{n-k}=\sum_{j=0}^{q} \sum_{k=0}^{n}(-1)^{j+k} G_{p+1}(q-j, n-k)+\sum_{k=0}^{n}(-1)^{k+q+1}
$$

and hence our assertion follows.

Separating multiple zeta value of largest weight $p+q+n+1$ from both sides of (3), we obtain the following corollary.

Corollary 5.4 Suppose that $n, p$ are positive integers. Then for integer $q$ with $0 \leq q \leq n$, we have

$$
\begin{aligned}
& \sum_{|\alpha|=p+q+n}\left(\begin{array}{c}
\alpha_{p} \\
q+1
\end{array}\right) \zeta\left(\alpha_{1}, \ldots, \alpha_{p-1}, \alpha_{p}+1\right)=G_{p+1}(q, n) \\
= & \sum_{k=q+1}^{n+1}\left(\begin{array}{c}
k-1 \\
q
\end{array}\right) \sum_{|\beta|=q+n+1} \zeta\left(\beta_{1}, \ldots, \beta_{k-1}, \beta_{k}+p\right) .
\end{aligned}
$$

Corollary 5.5(Arakawa \& Kaneko, 1999; Ohno, 1999) The identity (4) holds.

Remark Here is another identity concerning weighted sums of multiple zeta values (Chung \& Eie, 2017):

$$
\begin{aligned}
& \sum_{|\alpha|=n+p} \alpha_{p} \zeta\left(\alpha_{1}, \ldots, \alpha_{p-1}, \alpha_{p}+1\right) \\
= & \zeta(n+p+1)+\sum_{1 \leq \ell_{1}<\ell_{2}} \frac{1}{\ell_{1} \ell_{2}^{p}} \sum_{\lambda \vdash n} \mu_{\lambda}^{-1}\left(\widetilde{h}_{\lambda_{1}} \widetilde{h}_{\lambda_{2}} \cdots \widetilde{h}_{\lambda_{g}}-h_{\lambda_{1}}^{\prime} h_{\lambda_{2}}^{\prime} \cdots h_{\lambda_{g}}^{\prime}\right),
\end{aligned}
$$

where

$$
\widetilde{h}_{m}=\sum_{\ell=\ell_{1}}^{\ell_{2}} \frac{1}{\ell^{m}} \quad \text { and } \quad h_{m}^{\prime}=\sum_{\ell=\ell_{1}}^{\ell_{2}-1} \frac{1}{\ell^{m}} .
$$

So that we have another expression of $\zeta^{*}\left(\{1\}^{n}, p+1\right)$ :

$$
\begin{aligned}
& \zeta^{*}\left(\{1\}^{n}, p+1\right) \\
= & \zeta(n+p+1)+\sum_{1 \leq \ell_{1}<\ell_{2}} \frac{1}{\ell_{1} \ell_{2}^{p}} \sum_{\lambda \vdash n} \mu_{\lambda}^{-1}\left(\widetilde{h}_{\lambda_{1}} \widetilde{h}_{\lambda_{2}} \cdots \widetilde{h}_{\lambda_{g}}-h_{\lambda_{1}}^{\prime} h_{\lambda_{2}}^{\prime} \cdots h_{\lambda_{g}}^{\prime}\right) .
\end{aligned}
$$

\section{Conclusion}

This paper establishes two main results (Theorem 1.2 and Theorem 1.3) that derives many extensions and generalizations of sum formula of multiple zeta values. The proof of sum formula given by A. Granville (Granville, 1997) used generating functions. Our approach is based on the iterated integral representation (Drinfel'd integral) of multiple zeta values with additional factors or its deformation. A number of multiple-zeta-values-identities (allied infinite sums) have been shown directly along this direction. We hope these ideas will interest an array of scholars from. 


\section{Fund}

The corresponding author Chung was fully supported by Fuzhou University grant GXRC-18049: 0030-510649.

\section{Acknowledgment}

The authors thank the anonymous referees for comments which improved the quality of this paper.

\section{References}

Arakawa, T., \& Kaneko, M. (1999). Multiple zeta values, poly-Bernoulli numbers, and related zeta functions. Nagoya Math. J., 153, 189-209. https://doi.org/10.1017/S0027763000006954

Borwein, J. M., Bradley, D. M., Broadhurst, D. J., \& Lisoněk, P. (2001). Special values of multiple polylogarithms. Trans. Amer. Math. Soc., 353(3), 907-941.

Chen, K.-W., Chung, C.-L., \& Eie, M. (2016). Sum formulas and duality theorems of multiple zeta values. J. Number Theory, 158, 33-53. https://doi.org/10.1016/j.jnt.2015.06.014

Chung, C.-L., \& Eie, M. (2017). Some Variations of Multiple Zeta Values. Rocky Mountain J. Math., 47(7), $2107-2131$.

Drinfel'd, V. G. (1991). On quasitriangular quasi-Hopf algebras and on a group that is closely connected with Gal( $\overline{\mathbb{Q}} / \mathbb{Q})$, Leningrad Math. J., 2(4), 829-860.

Eie, M. (2009). Topics in Number Theory. Monographs in Number Theory vol.2. World Scientific Publishing Co. Pte. Ltd. https://doi.org/10.1142/7036

Eie, M., Liaw, W.-C. \& Ong, Y. L. (2009). A restricted sum formula among multiple zeta values, J. Number Theory, 129, 908-921. https://doi.org/10.1016/j.jnt.2008.07.012

Eie, M., \& Lee, T.-Y. (2016). Identities among restricted sums of multiple zeta values, J. Number Theory, 164, $208-222$. https://doi.org/10.1016/j.jnt.2015.11.016

Granville, A. (1997). A decomposition of Riemann's zeta-function, in “Analytic Number Theory”, Y. Motohashi (ed,), London Math. Soc. Lect. Note Ser., 247, Cambridge Univ. Press, 95-101.

MacDonald, I. G. (1995). Symmetric Functions and Hall Polynomials (2nd ed.). Clarendon Press.

Ohno, Y. (1999). A generalization of the duality and sum formulas on the multiple zeta values, J. Number Theory, 74, 39-43. https://doi.org/10.1006/jnth.1998.2314

Stanley, R. P. (1999). Enumerative Combinatorics vol. 2. Cambridge University Press. https://doi.org/10.1017/CBO9780511609589

\section{Copyrights}

Copyright for this article is retained by the author(s), with first publication rights granted to the journal.

This is an open-access article distributed under the terms and conditions of the Creative Commons Attribution license (http://creativecommons.org/licenses/by/4.0/). 